\title{
REGULAR SEMIGROUPS SATISFYING CERTAIN CONDITIONS ON IDEMPOTENTS AND IDEALS
}

\author{
BY
}

\section{MARIO PETRICH}

\begin{abstract}
The structure of regular semigroups is studied (1) whose poset of idempotents is required to be a tree or to satisfy a weaker condition concerning the behavior of idempotents in different $\mathfrak{D}$-classes, or (2) all of whose ideals are categorical or satisfy a variation thereof. For this purpose the notions of $D$ majorization of idempotents, where $D$ is a $D$-class, $D$-majorization, $D$-categorical ideals, and completely semisimple semigroups without contractions are introduced and several connections among them are established. Some theorems due to $G$. Lallement concerning subdirect products and completely regular semigroups and certain results of the author concerning completely semisimple inverse semigroups are either improved or generalized.
\end{abstract}

1. Introduction. An arbitrary regular semigroup $S$ has at least two outstanding features: (1) may have a very complicated structure, (2) abounds in idempotents. For these two reasons, it is natural to impose suitable restrictions on the partially ordered set $E_{S}$ of idempotents of $S$ in order to obtain various classes of regular semigroups more susceptible to a treatment which might eventually lead to a complete determination of its structure. Another type of restriction is a statement concerning different kinds of ideals of $S$. Such restrictions are sometimes expressed by means of Green's relations. As long as these restrictions are very strong, they lead to a complete determination of the structure (mod, e.g., semilattices or groups) of the semigroup satisfying them, witness: completely 0-simple semigroups (see [1], [9]), trees of completely 0-simple semigroups [8], simple regular $\omega$-semigroups $([5],[10])$, etc.

In addition to the frequent hypothesis that our semigroup be completely semisimple, we impose restrictions of either type mentioned above with the following variations: (1) the partially ordered set $E_{S}$ is required to be a tree or to satisfy a condition concerning the behavior of idempotents in different $\mathscr{D}$-classes of $S$, (2) all ideals are required to be categorical or satisfy a modified version thereof involving $\mathfrak{D}$-classes of $S$. With suitable restrictions on a regular semigroup, we are able to establish several connections among these conditions, and in some instances, characterize the semigroups in question in terms of subdirect products

Received by the editors May 10, 1970.

AMS 1970 subject clas sifications. Primary $20 \mathrm{M} 10$.

Key words and phrases. Completely regular semigroup, categorical ideal, completely semisimple semigroup, primitive regular semigroup, subdirect products, tree, normal band of groups, $D$-class, extensions, partial homomorphism. 
of (general or special) completely 0 -simple semigroups, or give their complete structure. The frequent change of hypotheses strongly suggests that many of our results are not the best possible. This opens a great number of problems particularly concerning subdirect products of 0 -bisimple regular semigroups.

Summary. We begin in $\$ 2$ by considering the relationship of two regular $\mathscr{D}$. classes $A$ and $B$ of an arbitrary semigroup $S$ in terms of the behavior of their idempotents and functions from $B$ into $A$ satisfying certain multiplicative conditions. The principal idea here is a generalization of the development first expounded by Lallement [6, Chapitre II, \$3] for the case of a completely semisimple semigroup. We introduce two binary relations on the set of regular $\mathscr{D}$-classes of $S$ and the notions of $C$-and $\sigma$-majorization, which are then used throughout the paper. Making a free use of the results in the author's paper [14], the main result of this section is a connection between the function $\chi_{A}$ introduced in [14] and $A$ majorization (a condition on idempotents) for a regular semigroup $S$. A number of lemmas are stated in a general setting and seem to be of independent interest.

In $\$ 3$ we specialize the situation by further restricting the condition on the functions $\chi_{D}$ for a regular semigroup $S$, and deduce a slight improvement of a theorem due to Lallement $[6,2: 12,2.17]$ concerning subdirect products of completely 0-simple semigroups, giving a simpler proof. This is further specialized in $\$ 4$ by considering completely regular semigroups, the main hypothesis always being $D$-majorization. Again we improve a theorem of Lallement [6, 2.21-2.23], giving several characterizations of the class of completely regular semigroups satisfying $\mathfrak{D}$-majorization.

We introduce in $\$ 5$ the concept of a $\sigma$-categorical ideal, and for a certain class of regular semigroups $S$ show that $\mathfrak{D}$-majorization implies that all ideals of $S$ are $\mathscr{D}$-categorical and that if $E_{S}$ is also a tree then all ideals are categorical. In order to establish a partial converse of these statements, we consider in $\$ 6$ a more general situation. Indeed, we introduce the notion of a completely semisimple semigroup without contractions and give simple necessary and sufficient conditions in order that an extension $V$ of a primitive regular semigroup $S$ without contractions be determined by a partial homomorphism. This is used in $\$ 7$ to obtain a partial converse of some statements in $\$ 5$. Several results in $\$ \$ 5-7$ have been established for inverse semigroups in the author's paper [15].

Notation. We follow the notation and terminology used in [1] with a few minor exceptions. The following is a list of frequently used notation. If $A$ is a nonempty subset of a semigroup $S$, then $E_{A}$ denotes the partially ordered set of idempotents contained in $A$, where $e \leq f \Leftrightarrow e=e f=f e$. To avoid cumbersome exceptions, we often suppose that the semigroup $S$ under consideration has a zero, which is denoted by $S=S^{0}$; usually an obvious modification yields the desired statement for the case without zero and will not be explicitly stated. For 
sets $A$ and $B$, we write $A \backslash B=\{a \in A \mid a \notin B\}$, and do not distinguish between an element and the set consisting of that element alone. The set of nonzero elements of a semigroup $S$ with zero is denoted by $S^{*}$. If $D$ is a $D$-class of $S$, then $D^{0}$ denotes the groupoid defined on the set $D \cup 0$ where $0 \notin D, 0$ acts as the zero, and all products of elements of $D$ that, in $S$, fall outside of $D$ are set equal to 0 . If $\alpha$ is a homomorphism of $S$, ker $\alpha$ is the congruence on $S$ induced by $\alpha$. We use the following notation frequently:

$$
M_{e}=\left\{f \in E_{S} \mid f \leq e\right\} \text { where } e \in E_{S} .
$$

2. Conditions on idempotents of regular $\mathscr{D}$-classes. We will establish here a number of auxiliary statements mainly concerning two regular $D$-classes of an arbitrary semigroup. These results are fundamental for a large portion of the paper.

Throughout this section, $A$ and $B$ denote regular $D$-classes of an arbitrary semigroup $S=S^{0}$.

Lemma 2.1. If $(A B \cup B A) \cap A \neq \varnothing$, then there exist $e \in E_{A}$ and $f \in E_{B}$ such that $e \leq f$.

Proof. Let $a \in A, b \in B$ and suppose that $a b \in A$. Let $f \in E_{B}$ be such that $b=b f$, and let $x=a b, x^{\prime}$ be an inverse of $x, e=f x^{\prime} x$. Then

$$
\begin{aligned}
& e^{2}=\left(f x^{\prime} x\right)\left(f x^{\prime} x\right)=f x^{\prime}(x f) x^{\prime} x=f x^{\prime} x x^{\prime} x=f x^{\prime} x=e, \\
& e f=\left(f x^{\prime} x\right) f=f x^{\prime}(x f)=f x^{\prime} x=e=f e,
\end{aligned}
$$

so that $e \leq f$. Further,

$$
x=x\left(x^{\prime} x\right)=a b x^{\prime} x=a(b f) x^{\prime} x=a b\left(f x^{\prime} x\right)=x e
$$

which together with $e=\left(f x^{\prime}\right) x$ implies that $e \mathcal{L} x$ and thus $e \in A$. The case $b a \in A$ is analogous.

It follows from [2, Théorème 2] that under the hypothesis of the lemma, for any $g \in E_{B}$, we have $M_{f} \cap E_{A} \cong M_{g} \cap E_{A}$ as partially ordered sets. We thus introduce the following notation:

$A \leq B$ means that for some (and thus any) $f \in E_{B}, M_{f} \cap E_{A} \neq \varnothing$,

$A \preceq B$ means that for some (and thus any) $f \in E_{B}$, the poset $M_{f} \cap E_{A}$ has a greatest element, to be denoted by $\bar{f}$.

The relations $\leq$ and $\preceq$ are defined on the set $\Delta$ of all regular $\mathcal{D}$-classes of $S$. It follows from the definition that both $\leq$ and $\preceq$ are reflexive and that $\leq$ is also transitive. In a simple regular $\omega$-semigroup with $D_{\text {-classes }} D_{1}, D_{2}, \ldots, D_{n}$ where $n>1$, we have $D_{i} \preceq D_{j}$ for any $1 \leq i, j \leq n$ showing that $\preceq$ and thus also $\leq$ need not be antisymmetric, see [5] or [10]. In fact, $\leq$ need not even be transitive. For take the set $T=\{1,2,3\}$, and transformations $\alpha=\left(\begin{array}{lll}1 & 2 & 3 \\ 1 & 2 & 3\end{array}\right), \beta=\left(\begin{array}{lll}1 & 2 & 3 \\ 1 & 1 & 3\end{array}\right)$, 
$\gamma=\left(\begin{array}{ll}1 & 23 \\ 1 & 1\end{array}\right), \delta=\left(\begin{array}{ll}1 & 23 \\ 2 & 22\end{array}\right) ;$ then $D_{\gamma}=D_{\delta} \preceq D_{\beta}, D_{\beta} \preceq D_{a}$, but $D_{\delta} \npreceq D_{a}$.

We will have many occasions to consider various functions from $B$ into $A$ which have certain multiplicative properties. It is then convenient to introduce the following notation. Let $\phi: B \rightarrow A$ be a function and $a \in A, b, b^{\prime} \in B$ be arbitrary elements. We will say that $\phi$ satisfies condition $(\alpha)$, resp. $(\beta)$, etc., if

(a) $a b \in A \Rightarrow a b=a(b \phi), b a \in A \Rightarrow b a=(b \phi) a$;

( $\beta) E_{B} \phi \subseteq E_{A}$;

( $\gamma) a b \in A$ or $a(b \phi) \in A \Rightarrow a b=a(b \phi), b a \in A$ or $(b \phi) a \in A \Rightarrow b a=(b \phi) a$;

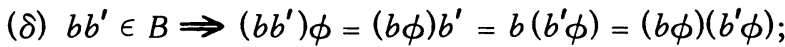

and if $C$ is a regular $\mathscr{D}$-class of $S$ and $\psi: C \rightarrow A$ (for $C=B$, take $\psi=\phi$ ),

( $\epsilon) b \in B, c \in C, b c \in A \Rightarrow b c=(b \phi) c=b(c \psi)=(b \phi)(c \psi)$.

The purpose of the next two lemmas is to construct a function from $B$ to $A$ satisfying $(\alpha)$ and $(\beta)$ provided that $A \preceq B$.

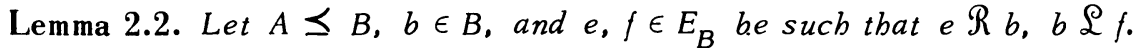
Then $\bar{e} b=b \bar{f} \in A$ and this element is independent of the choice of $e \in R_{b}, f \in L_{b}$.

Proof. Recall that $\bar{e}$ is the greatest element of $M_{e} \cap E_{A}$. To prove the first statement, by symmetry, it suffices to show that $b \bar{f}=\bar{e} b \bar{f} \in A$.

Since $b \mathfrak{L} f$ and $\mathcal{L}$ is a right congruence, we obtain $b \bar{f} \mathfrak{\mathscr { f }} f \bar{f}=\bar{f}$ so $b \bar{f} \in A$. Let $g$ be an idempotent such that $g R b \bar{f}$; then $b \bar{f}=g(b \bar{f})$ and $g=(b \bar{f}) u$ for some $u \in S$. Consequently,

$$
(g e)^{2}=(b \bar{f} u) e(b \bar{f} u) e=b \bar{f} u(e b) \bar{f} u e=(b \bar{f} u)^{2} e=g e,
$$

so ge $\in E_{S}$. Further, $g=b \bar{f} u=g(e b) \bar{f} u$ implies that $g$ e $R g$, so ge $R b \bar{f}$. Since $b \bar{f} \in A$, it follows that $g e \in A$. Thus $g e \in E_{A}$ and $g e=b \bar{f} u e=(e b) \bar{f} u e=e g e \mathrm{im}-$ plies that $g e \leq e$. But then $g e \leq \bar{e}$ and we obtain

$$
b \bar{f}=g(b \bar{f})=g(e b) \bar{f}=(g e) b \bar{f}=\bar{e}(g e) b \bar{f}=\bar{e} b \bar{f} .
$$

The second statement of the lemma follows from the first since there is no connection between $e$ and $f$.

Because of this lemma, if $A \leq B$ we are able to define a function $\eta_{B A}: B$ $\rightarrow A$ as follows:

$$
\eta_{B A}: b \rightarrow \bar{e} b=b \bar{f} \quad \text { where } e \Re b, b \mathfrak{\&}(b \in B) .
$$

Lemma 2.3. If $A \preceq B$, then $\eta_{B A}$ satisfies $(\alpha)$ and $(\beta)$.

Proof. Let $a \in A, b \in B$, and $e \in E_{B}$ be such that $a b \in A, e \Re b$. Then $a b$ $=a(e b)=(a e) b$ and $a\left(b \eta_{B A}\right)=a(\bar{e} b)=(a \bar{e}) b$; thus to prove that $a b=a(b \phi)$, it suffices to show that $a e=a \bar{e}$. First note that $e \Re b$ implies $a e R a b$ so that ae $\epsilon A$. Letting $t$ be an inverse of $a e$, and $b=e t a e$, we obtain $b \in E_{S}, b \leq e$, 
$b \mathfrak{Q} a e$ and hence $b \leq \bar{e}$. Consequently,

$$
a e=a b=a(b \bar{e})=(a b) \bar{e}=(a e) \bar{e}=a \bar{e}
$$

as desired. Therefore $a b=a\left(b \eta_{B A}\right)$; symmetrically $b a \in A$ implies $b a=\left(b \eta_{B A}\right) a$. Since for any $e \in E_{B}$, we have $e \eta_{B A}=\bar{e}$, it follows that $E_{B} \eta_{B A} \subseteq E_{A}$. Q.E.D.

The next eight lemmas treat the relationships among the conditions $A \leq B$ and $A \preceq B$, the function $\eta_{B A}$, and functions $\phi$ susceptible to satisfying certain of the conditions $(\alpha)-(\epsilon)$.

Lemma 2.4. If $A \leq B$ and there exists $\phi$ satisfying $(\alpha)$ and $(\beta)$, then $A \preceq B$.

Proof. For any $e \in E_{B}$, we have $e \phi \in E_{A}$. If $f \in M_{e} \cap A$, then $f=f e=e f$ so that $f=(e \phi) f=f(e \phi)$ and thus $f \leq e \phi$. Hence $e \phi$ is the greatest element of $M_{e}$ $\cap A$.

Lemma 2.5. If $A \preceq B$ and $A$ is also a I-class of $S$, then $\eta_{B A}$ satisfies $(\gamma)$.

Proof. Since by 2.3 the function $\eta_{B A}$ satisfies $(\alpha)$, by symmetry, it suffices to show that for $a \in A, b \in B$, the inclusion $a\left(b \eta_{B A}\right) \in A$ implies $b a \in A$. Indeed, for $f \in E_{B}$ such that $b \mathfrak{\&}$, we obtain

$$
J(a)=J\left(a\left(b \eta_{B A}\right)\right)=J(a b \bar{f}) \subseteq J(a b) \subseteq J(a)
$$

so that $a b \in A$.

Lemma 2.6. If $A \preceq B$ and $B^{0}$ is completely 0-simple, then $\eta_{B A}$ satisfies $(\delta)$.

Proof. For $b, b^{\prime}, b b^{\prime} \in B$, if $e \in E_{B}$ satisfies $e \Re b$, then also $e R b b^{\prime}$ since $B^{0}$ is completely 0 -simple. Hence

$$
\left(b b^{\prime}\right) \eta_{B A}=\bar{e}\left(b b^{\prime}\right)=(\bar{e} b) b^{\prime}=\left(b \eta_{B A}\right) b^{\prime}
$$

and thus, by $(a)$, also, $\left(b \eta_{B A}\right) b^{\prime}=\left(b \eta_{B A}\right)\left(b^{\prime} \eta_{B A}\right)$ since $\left(b b^{\prime}\right) \eta_{B A} \in A$. Analogously, $\left(b b^{\prime}\right) \eta_{B A}=b\left(b^{\prime} \eta_{B A}\right)$. Q.E.D.

Lemmas 2.5 and 2.6 cease to be valid if both $A$ and $B$ are supposed to be only regular. For example, take again a simple regular $\omega$-semigroup $S$ which is not bisimple. In the notation of [5], letting $e_{1}>e_{2}>\cdots>e_{k}$ be the poset of all idempotents of the chain of groups $G$, we specify $A=D_{\left(0, e_{1}, 0\right)}, B=D_{(0, e, 2,0)}$. A straightforward calculation shows that, for $a=\left(0, e_{1}, 0\right), b=\left(0, e_{2}, 0\right), b^{\prime}=$ $\left(1, e_{2}, 0\right)$, we have $a \in A, b, b^{\prime} \in B$ and (1) $a\left(b \eta_{B A}\right) \in A$ but $a b \notin A$ so that $(\gamma)$ fails, (2) $b b^{\prime} \in B$ but $\left(b \eta_{B A}\right)\left(b^{\prime} \eta_{B A}\right) \neq\left(b \eta_{B A}\right) b^{\prime}$ so that $(\delta)$ fails.

Lemma 2.7. If $A^{0}$ is completely 0 -simple and $\phi$ is a function satisfying $(\alpha)$, then $A \preceq B$ and $\phi=\eta_{B A}$.

Proof. If $e \in E_{B^{\prime}} f, g \in E_{A}$ satisfy $e \geq f, e \geq g$, then, by $(a), f=(e \phi) f=$ 
$f(e \phi)$ which in a completely 0 -simple semigroup implies that $e \phi=f$ and, analogously, $e \phi=g$. Consequently, $f=g$ and $A \preceq B$. Let $b \in B$ and $e, f \in E_{B}$ be such that $e \Re b, b \mathfrak{\&}$. Then $b \eta_{B A}=\bar{e} b \in A$ which by $(a)$ implies $\bar{e} b=\bar{e}(b \phi)$ so that $b \eta_{B A}=\bar{e}(b \phi)$ and, symmetrically, $b \eta_{B A}=(b \phi) \bar{f}$. A simple computation using a Rees matrix representation of $A^{0}$ shows that the last two equations imply $b \phi=b \eta_{B A}$.

Lemma 2.8. If $A \leq B$ and there exists a function $\phi$ satisfying $(\alpha)$ and $(\delta)$, then $A \preceq B$ and $\phi=\bar{\eta}_{B A}$.

Proof. First note that $(\delta)$ implies $(\beta)$. Hence by 2.4 we have that $A \preceq B$ and by its proof that, for any $e \in E_{B}$, e $\phi$ is the greatest element of $M_{e} \cap E_{A}$. Consequently, $e \phi=\bar{e}=e \eta_{B A}$. For any $b \in B$, there is $e \in E_{B}$ such that $e \Re b$, and hence by $(\delta)$,

$$
b \phi=(e b) \phi=(e \phi) b=\bar{e} b=b \eta_{B A} .
$$

Lemma 2.9. If $A \preceq B, A \preceq C, b \in B, c \in C, b c \in A$, then $\eta_{B A}$ and $\eta_{C A}$ satisfy $(\epsilon)$.

Proof. For $e \in E_{B}$, $e \Re b$, by $(\alpha)$ we obtain

$$
b c=(e b) c=\left(e \eta_{B A}\right) b c=\bar{e}(b c)=(\bar{e} b) c=\left(b \eta_{B A}\right) c=\left(b \eta_{B A}\right)\left(c \eta_{C A}\right) .
$$

and dually $b c=b\left(c \eta_{C A}\right)$.

Lemma 2.10. If $A \preceq B, B \preceq C, A \leq C$, and $A^{0}$ and $B^{0}$ are completely 0 simple, then $\eta_{C B} \eta_{B A}=\eta_{C A}$.

Proof. For $c \in C$, e $\in E_{C}$ such that $e R c$, let $e_{B}$ be the greatest element of $M_{e} \cap B$ and $e_{A}$ be the greatest element of $M_{e_{B}} \cap A$. Using ( $\delta$ ) (see 2.6), we obtain

$$
c \eta_{C B} \eta_{B A}=\left(e_{B} c\right) \eta_{B A}=\left(e_{B}\left(e_{B} c\right)\right) \eta_{B A}=\left(e_{B} \eta_{B A}\right) e_{B} c=e_{A} e_{B} c=e_{A} c .
$$

On the other hand, $e \geq e_{B} \geq e_{A}$ and $A^{0}$ completely 0-simple imply that $e_{A}$ is the only element in $M_{e} \cap A$, so that $c \eta_{C A}=e_{A} c$ and the desired equality follows.

Lemma 2.11. If $A=J_{a}$ and $B=J_{b}$, then $A \leq B$ if and only if $J(a) \subseteq J(b)$.

Proof. If $A \leq B$, then for some $e \in E_{A}, f \in E_{B}$ we have $e \leq f$ so that $e \epsilon$ $J(f)$ and thus $J(a)=J(e) \subseteq J(f)=J(b)$. Conversely, suppose that $J(a) \subseteq J(b)$. For any $e \in E_{A}$ we have $e=x b y$ for some $x, y \in S$. Then $e=e x b y$ and thus

$$
J(e)=J(e x b y) \subseteq J(e x b) \subseteq J(e x) \subseteq J(e)
$$

which implies that $e x,(e x) b \in A$. Consequently, $A B \cap A \neq \varnothing$ and 2.1 implies 
$A \leq B$

As a consequence we have that if $D=I$ in a regular semigroup $S$ then the relation $\leq$ agrees with the usual partial ordering of $\mathcal{I}$-classes defined by $J_{a} \leq J_{b}$ if and only if $J(a) \subseteq J(b)$.

By $T_{A}$ denote the trace of $A$ and let $\chi_{A}$ be the homomorphism of $S$ into the translational hull $\Omega\left(T_{A}\right)$ of $T_{A}$ constructed in $[14, \$ 3]$. In particular,

$$
\operatorname{ker} \chi_{A}=\left(\operatorname{ker} \chi_{A} \pi_{\mathrm{P}}\right) \cap\left(\operatorname{ker} \chi_{A} \pi_{\Lambda}\right)
$$

where

ker $\chi_{A} \pi_{\mathrm{P}}=\{(s, t) \mid$ if $a \in A$ and either as $\mathcal{R} a$ or at $R a$, then $a s=a t\}$ and for ker $\chi_{A} \pi_{A}$ there is a dual expression.

Recall that an (ideal) extension $V$ of a semigroup $S$ is dense if the equality relation on $V$ is the only congruence on $V$ whose restriction to $S$ is the equality on $S$ (see, e.g., [3]). Also recall the notation $\pi_{s}=\left(\lambda_{s}, \rho_{s}\right)$.

Proposition 2.12. If $A$ is both a $\mathfrak{D}$ - and $\mathrm{J}$-class of a regular semigroup $S=$ $S^{0}$, then $S_{\chi_{A}}$ is a dense extension of the 0-bisimple regular semigroup $A \chi_{A} \cup \pi_{0}$.

Proof. Let $F=A \chi_{A} \cup \pi_{0}$. If $a \in A, s \in S$, then

$$
\text { as } \in J(a)=J_{a} \cup I(a)=A \cup I(a)
$$

so that $\left(a \chi_{A}\right)\left(s \chi_{A}\right)=(a s) \chi_{A} \in F$, and dually $\left(s \chi_{A}\right)\left(a \chi_{A}\right) \in F$, which proves that $F$ is an ideal of $S_{\chi_{A}}$. Since $A$ is a regular D-class and $A_{\chi_{A}} \cup \pi_{0}=F$ is an ideal of $S \chi_{A}$, it follows that $A \chi_{A}$ is a regular $D_{\text {-class of }} S_{\chi_{A}}$, so $F$ is a 0 -bisimple regular semigroup. To prove that the extension is dense, we suppose that, for $s, t \in S$,

$$
\left(s \chi_{A}\right)\left(a \chi_{A}\right)=\left(t \chi_{A}\right)\left(a \chi_{A}\right), \quad\left(a \chi_{A}\right)\left(s \chi_{A}\right)=\left(a \chi_{A}\right)\left(t \chi_{A}\right) \quad(a \in A) .
$$

Then $(s a, t a),(a s, a t) \in \operatorname{ker} \chi_{A}(a \in A)$. Let $a \in A$ and suppose as $R a$. For $e \in E_{S}$ such that $e R a$, we get $e a=a$ and $e R$ as so that $e R e(a s)$. Since $(a s, a t) \in \operatorname{ker}\left(\chi_{A} \pi_{\mathrm{p}}\right)$, it follows that eas = eat and hence as =at. Symmetrically,

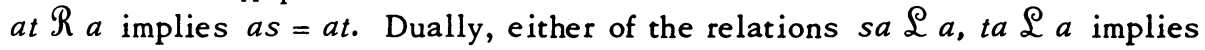
$s a=t a$. But then $(s, t) \in \operatorname{ker} \chi_{A}$ which proves that $s \chi_{A}=t \chi_{A}$, which by $[3,3.7]$ implies that $S_{\chi_{A}}$ is a dense extension of $F$.

The conclusion of 2.12 fails for all but one $D$-class of a simple but not bisimple regular $\omega$-semigroup $S$ (again by a straightforward computation using the representation in [5] or [10]).

Corollary 2.13. Every regular semigroup $S=S^{0}$ in which $\mathfrak{D}=\mathscr{G}$ is a subdirect product of dense extensions of 0-bisimple regular semigroups.

Proof. This follows from [14, Proposition 2] and 2.12. 
We are now interested in the case $S_{\chi_{A}}=A \chi_{A} \cup \pi_{0}$ for a regular semigroup $S$, for if this holds for all $D$-classes, then $S$ is a subdirect product of 0 -bisimple regular semigroups $A \chi_{A} \cup \pi_{0}$.

Lemma 2.14. $B \chi_{A} \subseteq A \chi_{A}$ if and only if there exists a function $\phi$ satisfying $(\gamma)$.

Proof. Necessity. The hypothesis implies that for every $b \in B$ there exists $a \in A$ such that $b \chi_{A}=a \chi_{A}$._Since $\left.\chi_{A}\right|_{A}$ is one-to-one, $a$ is unique and we write $\phi: b \rightarrow a=\bar{b}$. Hence $\lambda^{b}=\lambda^{\bar{b}}$ and thus for every $(a ; i, \mu) \in T_{A}$, we have

$$
b H_{i 1}=H_{j 1} \Leftrightarrow \bar{b} H_{i 1}=H_{j 1} \Rightarrow r_{j}^{\prime} b r_{i}=r_{j}^{\prime} \bar{b} r_{i} .
$$

Let $a \in A$, then $a \in H_{i \mu}$ for some $i$ and $\mu$, and thus $a=r_{i} u$ for some $u \in S$. Suppose next that $b a \in A$. Then $b a=b r_{i} u \in A$ and since $b r_{i} u R b r_{i}$, it follows that $b r_{i} \in A$. Hence $b H_{i 1}=H_{j 1}$ for some $j$ and (1) implies that $\bar{b} H_{i 1}=H_{j 1}$ and $r_{j}^{\prime} b r_{i}=$ $r_{j}^{\prime} \bar{b} r_{i}$. By virtue of $r_{j} r_{j}^{\prime}=e_{j} R b r_{i}$ and $e_{j} \Re \bar{b} r_{i}$, we obtain

$$
b a=b\left(r_{i} u\right)=e_{j}\left(b r_{i}\right) u=r_{j}\left(r_{j}^{\prime} b r_{i}\right) u=r_{j}\left(r_{j}^{\prime} \bar{b} r_{i}\right) u=e_{j}\left(\bar{b}_{i}\right) u=\bar{b}\left(r_{i} u\right)=\bar{b} a .
$$

Conversely, if $\bar{b} a \in A$, an analogous proof shows that then $\bar{b} a=b a$. This establishes the second part of $(\gamma)$ for the function $\phi$; the first part follows similarly from $\rho^{b}=\rho^{\bar{b}}$.

Sufficiency. For any $b \in B$, again writing $\bar{b}=b \phi$, if $b H_{i 1}=H_{j 1}$, then $\bar{b} r_{i}=$ $b r_{i} \in H_{j 1}$ and hence $r_{j}^{\prime} b r_{i}=r_{j}^{\prime} \bar{b} r_{i} \in H_{11}$. Similarly, if $\bar{b} H_{i 1}=H_{j 1}$, then $r_{j}^{\prime} b r_{i}=$ $r_{j}^{\prime} \bar{b} r_{i} \in H_{11}$. Consequently, $\lambda^{b}=\lambda^{\bar{b}}$, and dually $\rho^{b}=\rho^{\bar{b}}$ and thus $b \chi_{A}=\bar{b} \chi_{A}$.

The following concept will prove very useful.

Definition 2.15. Let $C$ be a nonempty subset of a semigroup $S$. Then $S$ satisfies $C$-majorization if, for any $e \in E_{S}$, either $M_{e} \cap C=\varnothing$ or $M_{e} \cap C$ has a greatest element.

Theorem 2.16. Let $S=S^{0}$ be a regular semigroup and let $A$ be a D-class of $S$. If $S \chi_{A}=A \chi_{A} \cup \pi_{0}$, then $S$ satisfies $A$-majorization; the converse bolds if $A$ is also a I-class of $S$.

Proof. Suppose that $S_{\chi_{A}}=A \chi_{A} \cup \pi_{0}$ and let $B$ be an arbitrary $\mathscr{D}_{\text {-class of }}$ $S$. If $A \leq B$ does not hold, then, for every $e \in E_{B}$, we have $M_{e} \cap A=\varnothing$. If $A \leq B$, then $B \chi_{A} \subseteq A \chi_{A}$ since $\chi_{A}$ maps $B$ into a D-class of $A \chi_{A} \cup \pi_{0}$, and 2.14 implies the existence of a function $\phi: B \rightarrow A$ satisfying $(\gamma)$. Since $(\gamma)$ implies both $(\alpha)$ and $(\beta)$, by 2.4 we have that $A \preceq B$.

Now suppose that $A$ is also a $\mathcal{I}$-class of $S$ and that $S$ satisfies $A$-majoriza-

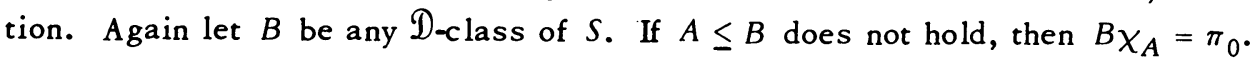
If $A \leq B$, then 2.5 implies that $\eta_{B A}$ satisfies $(\gamma)$ which by 2.14 yields $B \chi_{A} \subseteq$ $A \chi_{A}$. Consequently, $S \chi_{A}=A \chi_{A} \cup \pi_{0}$. 
We now introduce the notion which for the case $\sigma=\mathscr{D}$ will be a fundamental hypothesis for a great number of statements throughout the paper.

Definition 2.17. Let $\sigma$ be an equivalence relation on a semigroup $S$. Then $S$ satisfies $\sigma$-majorization if $S$ satisfies $C$-majorization for all equivalence classes $C$ of $\sigma$.

Corollary 2.18. Every regular semigroup $S=S^{0}$ satisfying $\mathscr{D}=\mathcal{I}$ and $\mathscr{D}-m a-$ jorization is a subdirect product of 0-bisimple regular semigroups.

Proof. Let $\Delta$ be an index set of $\mathscr{D}$-classes of $S$ different from 0 . Then [14, Proposition 2] says that the mapping $\chi: s \rightarrow\left(s \chi_{D}\right)_{D \in \Delta}$ is an isomorphism of $S$ into the direct product $\Pi_{D \in \Delta} \Omega\left(T_{D}\right)$ (we have omitted here the $\mathscr{D}_{\text {-class } 0} 0$ as we may). Consequently, $S$ is a subdirect product of semigroups $S_{\chi_{A}}, A \in \Delta$. Since for every $A \in \Delta$, by hypothesis $A$ is also a $\mathcal{G}$-class and $S$ satisfies $A$-majorization, 2.16 implies that $S_{\chi_{A}}=A \chi_{A} \cup \pi_{0}$ so that $S_{\chi_{A}}$ is a 0-bisimple regular semigroup:

Theorem 2.16 and its corollary have their obvious analogues for the case of a regular semigroup without zero. Indeed, if $A$ in 2.16 is the kernel of $S$, then $S_{\chi_{A}}=A \chi_{A}$ should be substituted for $S_{\chi_{A}}=A \chi_{A} \cup \pi_{0}$, otherwise the statement remains the same. Corollary 2.18 for the general case becomes

Corollary 2.19. Every regular semigroup satisfying $\mathfrak{D}=\mathscr{I}$ and $\mathfrak{D}$-majorization is a subdirect product of 0-bisimple and bisimple regular semigroups.

3. Subdirect products of completely 0 -simple semigroups. Recall that for any semigroup $S$, the inner part $\Pi(S)$ of the translational hull $\Omega(S)$ of $S$ is the ideal of $\Omega(S)$ consisting of all $\pi_{s}=\left(\lambda_{s}, \rho_{s}\right), s \in S$, where $\lambda_{s} x=s x, x \rho_{s}=x s$ for all $x \in S$ (see, e.g., [3]). We are interested here in conditions on a regular $\mathscr{D}$-class $D$ of a semigroup $S$ in order that $S_{\chi_{D}}=\Pi\left(T_{D}\right)$. As a consequence we deduce a slight improvement of an important theorem due to Lallement [6], giving a simpler proof.

Lemma 3.1. Let $D$ be a regular $D$-class of a semigroup $S=S^{0}$. Then $D \chi_{D}=$ $\Pi\left(T_{D}\right) \backslash \pi_{0}$ if and only if $D^{0}$ is a completely 0-simple semigroup.

Proof. Necessity. The function $\left.\chi_{D}\right|_{D}$ is a partial isomorphism of $D$ onto $\Pi\left(T_{D}\right) \backslash \pi_{0}$ since $\chi_{D}$ is a homomorphism of $S$ whose restriction to $D$ is one-toone. For $e, f \in E_{D}, e \leq f$ implies $e \chi_{D} \leq f \chi_{D}$ and, since $\Pi\left(T_{D}\right)$ is completely 0simple, we must have $e \chi_{D}=f \chi_{D}$. But then $e=f$, proving that every nonzero idempotent of $D^{0}$ is primitive. Since $D^{0}$ is 0-bisimple, the Rees theorem implies that $D^{0}$ is completely 0 -simple.

Sufficiency. For $s \in D$ with $s \in R_{j} \cap L_{\nu}$, let $\bar{s}=\left(r_{j}^{\prime} s q_{\nu}^{\prime} ; j, \nu\right)$; then 


$$
\begin{array}{rlrl}
\lambda^{s}(a ; i, \mu) & =\left(r_{k}^{\prime} s r_{i} a ; k, \mu\right) & & \text { if } s H_{i 1}=H_{k 1} \\
& =0 & & \text { otherwise, } \\
\lambda_{s}(a ; i, \mu) & =\left(r_{j}^{\prime} s q_{\nu}^{\prime} ; j, \nu\right)(a ; i, \mu)=\left(r_{j}^{\prime} s q_{\nu}^{\prime}\left(q_{\nu} r_{i}\right) a ; j, \mu\right) \\
& =\left(r_{j}^{\prime} s\left(q_{\nu}^{\prime} q_{\nu}\right) r_{i} a ; j, \mu\right) \\
& =\left(r_{j}^{\prime} s r_{i} a ; j, \mu\right) & \text { if } q_{\nu} r_{i} \in H_{11}, \\
& =0 & & \text { otherwise. }
\end{array}
$$

Now giving $D^{0}$ the same Rees matrix representation used in constructing $T_{D}$, we have $s=(g ; j, \nu), q_{\nu}=(b ; 1, \nu), r_{i}=(t ; i, 1)$ for some $g, b, t \in H_{11}$, and obtain

$$
\begin{aligned}
s r_{i} & =(g ; j, \nu)(t ; i, 1)=\left(g p_{\nu i} t ; j, 1\right), \\
q_{\nu} r_{i} & =(b ; 1, \nu)(t ; i, 1)=\left(b p_{\nu i} t ; 1,1\right),
\end{aligned}
$$

which implies $s H_{i 1}=H_{k 1} \Leftrightarrow q_{\nu} r_{i} \in H_{11} \Rightarrow k=j$. This together with (1) and (2) shows that $\lambda^{s}(a ; i, \mu)=\lambda_{\bar{s}}(a ; i, \mu)$. Consequently, $\lambda^{s}=\lambda_{\bar{s}}$ and a symmetric proof shows that also $\rho^{s}=\rho_{\bar{s}}$, so that $s \chi_{D}=\pi_{\bar{s}}$. It is easy to see that for $(g ; j, \nu) \in T_{D}$, letting $s=r_{j} g q_{\nu}$, we obtain $s \chi_{D}=\pi_{\bar{s}}=\pi_{(g ; j, \nu)}$. Therefore, $\chi_{D}$ maps $D$ onto $\Pi\left(T_{D}\right) \backslash \pi_{0}$.

Note that in the proof of necessity it is enough to assume that $D_{\chi_{D}} \subseteq$ $\Pi\left(T_{D}\right) \backslash \pi_{0}$.

Lemma 3.2. If $D$ is a D-class of a semigroup $S$ and $D^{0}$ is completely 0-simple, then $D$ is a -class of $S$.

Proof. Let $J$ be the $\mathscr{G}$-class of $S$ containing $D$. Then the principal factor $J^{0}$ is 0 -simple, and if it is not completely 0 -simple, by [1, Theorem 2.54], for every idempotent $e$ in $D, J^{0}$ contains a copy $B$ of the bicyclic semigroup with identity $e$. But then for some $f \in E_{B}$, we have $e>f$ and $e \mathscr{D} f$, implying that $D$ contains two comparable nonzero idempotents. This contradicts the hypothes is that $D^{0}$ is completely 0 -simple. Hence $J^{0}$ is completely 0 -simple and $D=J$.

Theorem 3.3. Let $D$ be a D-class of a regular semigroup $S=S^{0}$ with $D \neq 0$. Then $S_{\chi_{D}}=\Pi\left(T_{D}\right)$ if and only if $D^{0}$ is completely 0-simple and $S$ satisfies $D$ majorization.

Proof. Necessity. If for some $s \in D$ we have $s \chi_{D}=\pi_{0}$, then since $\chi_{D}$ is a homomorphism, we also have $D \chi_{D}=\pi_{0}$. If $D$ has only one element, say $x$, then $x$ is an idempotent and $x \chi_{D}=\pi_{0}$ is impossible, and if $D$ has more than one element, $D \chi_{D}=\pi_{0}$ contradicts the fact that $\chi_{D}$ restricted to $D$ is one-to-one. Hence $D \chi_{D} \subseteq \Pi\left(T_{D}\right) \backslash \pi_{0}$ and 3.1, together with the remark following it, implies that $D^{0}$ is completely 0 -simple, which in turn, again by 3.1 , implies that $D_{\chi_{D}}=$ 
$\Pi\left(T_{D}\right) \backslash \pi_{0}$. It follows that $S_{\chi_{D}}=\Pi\left(T_{D}\right)=D \chi_{D} \cup \pi_{0}$ which by 2.16 implies that $S$ satisfies $D$-majorization.

Sufficiency. Since $D^{0}$ is completely 0-simple, 3.1 implies that $D \chi_{D} \cup \pi_{0}=\Pi\left(T_{D}\right)$ and 3.2 that $D$ is also a $g$-class of $S$. Hence by 2.16 , we have $S \chi_{D}=D \chi_{D} \cup \pi_{0}$ $=\Pi\left(T_{D}\right)$.

Recall that a semigroup $S$ is said to be completely semisimple if each principal factor of $S$ is completely (0-)simple.

Theorem 3.4 (cf. $[6,2.12,2.17])$. The following conditions on a regular semigroup $S$ are equivalent.

(a) $S$ is completely semisimple and satisfies D-majorization.

(b) $S$ is completely semisimple and, for any elements $a, b \in S, J(a) \subseteq J(b)$ implies $D_{a} \leq D_{b}$ and the existence of a function $\phi: D_{b} \rightarrow D_{a}$ satisfying $(\alpha)$.

(c) $S$ is a subdirect product of completely (0-)simple semigroups.

The function $\phi$ in (b) is equal to $\eta_{D_{b} D_{a}}$ and satisfies $(\beta),(\gamma),(\delta)$, and the system of functions $\left\{\eta_{D_{b} D_{a}}\right\}$ satisfies $(\epsilon)$ and has the transitivity property in 2.10 .

Proof. (a) $\Rightarrow$ (b). This follows from 2.11 and 2.3.

(b) $\Rightarrow$ (a). This follows from 2.11 and 2.7.

(a) $\Rightarrow$ (c). It follows from 3.3 that $S$ is a subdirect product of completely

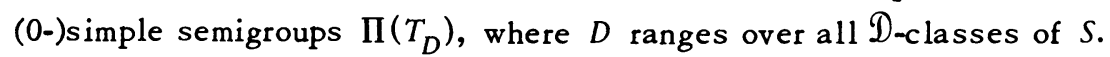

(c) $\Rightarrow($ a). Assume that $S$ is a subdirect product of completely (0-)simple semigroups $S_{\alpha}, a \in A$. Then $S$ is completely semisimple by [6, Lemma 2.14]. We suppose (as we may) that $S \subseteq \Pi_{a \in A} S_{a}$ and also that $e, f, g \in E_{S}$ have the properties $e \geq f, e \geq g, f \mathfrak{D} g$. Writing $e=\left(e_{a}\right), f=\left(f_{a}\right), g=\left(g_{a}\right)$, it follows that for every $a \in A, e_{\alpha} \geq f_{\alpha}, e_{\alpha} \geq g_{\alpha}, f_{\alpha}=0 \Leftrightarrow g_{\alpha}=0$. Hence if $f_{\alpha} \neq 0$, then $f_{\alpha} \leq e_{\alpha}$ implies $f_{\alpha}=e_{\alpha}$, and $g_{\alpha} \neq 0$ so $g_{\alpha} \leq e_{\alpha}$ implies $g_{\alpha}=e_{\alpha}$, hence $f_{\alpha}=g_{\alpha}$. Consequently, $f=\left(f_{a}\right)=\left(g_{\alpha}\right)=g$ which establishes $\mathscr{D}$-majorization.

When (b) holds, for $A=D_{a}$ and $B=D_{b}$, by 2.11 and 2.7 we obtain $A \preceq B$ and $\phi=\eta_{B A}$. Hence $\phi$ satisfies $(\gamma)$ by 2.5 and $(\delta)$ by $2.6((\delta)$ trivially implies $(\beta))$, and the system $\left\{\eta_{B A}\right\}$ has the required properties by 2.9 and 2.10 .

4. Completely regular semigroups. We will now apply some of the results in the preceding section to this class of semigroups, obtain additional characterizations and a construction of the semigroups in question. The results here supplement some of those of Lallement [6, Chapitre II, \$4]. Recall that a semigroup $S$ is called completely regular if for every $a \in S$ there exists $b \in S$ such that $a=$ $a b a, a b=b a$; equivalently, $S$ is a union of (pairwise disjoint) groups (see [1], [9]). $A$ band (idempotent semigroup) $B$ is called (left, resp. right) normal if it satisfies the identity $(a x y=a y x$, resp. $x y a=y x a)$ axya=ayxa (see, e.g., [17]). Note that in a completely regular semigroup $S$, the relation $\mathcal{D}$ is the smallest semilattice 
congruence on $S$ and each $D$-class is completely simple, so $S$ is a semilattice of completely simple semigroups (and conversely). In accordance with this terminology, we say that a semigroup $S$ is a normal band of groups if $\mathcal{H}$ is a congruence, $S / \mathcal{H}$ is a normal band, and every $\mathcal{H}$-class of $S$ is a group. The last requirement is equivalent to the fact that $S$ is completely regular. If $a$ is an element of a completely regular semigroup $S$, we denote by $a^{*}$ the identity of the maximal subgroup of $S$ containing $a$.

Theorem 4.1 (cf. [6, 2.22, 2.23]). The following conditions on a completely regular semigroup $S$ are equivalent.

(a) $S$ satisfies D-majorization.

(b) Botb $\mathscr{Q}$ and $R$ are congruences, $S / \mathcal{Q}$ is a right normal band, $S / R$ is a left normal band.

(c) $S$ is a normal band of groups.

(d) For any $e, f, g \in E_{S}, e \leq f$ implies $(e g)^{*} \leq(f g)^{*},(g e)^{*} \leq(g f)^{*}$.

(e) $S$ is a subdirect product of completely simple semigroups with a zero possibly adjoined.

Proof. (a) $\Rightarrow(b)$. Since $R$ is always a left congruence, it suffices to show that it is here a right congruence. Thus let $a, b, c \in S, a R b, b^{\prime}$ be an inverse of $b, f=b b^{\prime}, t$ be an inverse of $b c, D$ be the $\mathscr{D}$-class of $S$ containing $b c$. Then $b c t b b^{\prime} \in E_{D}$ and $f \geq b c t b b^{\prime}$. Also $a c, c b^{\prime} \in D$ and thus there exists $v \in D$ such that $a c v c b^{\prime} \in E_{D}$ since $D$ is completely simple. Now $a=b x$ for some $x \in S$ and thus $f a=b b^{\prime} a=b b^{\prime}(b x)=b x=a$ so that $f \geq a c v c b^{\prime}$. Consequently, the hypothesis implies that $b c t b b^{\prime}=a c v c b^{\prime}$ and hence

$$
b c=(b c) t(b c)=\left(b c t b b^{\prime}\right)(b c)=\left(a c v c b^{\prime}\right)(b c) \in a c S
$$

and, dually, $a c \in b c S$. Therefore, $a c R b c$ which proves that $R$ is a congruence.

Now let $a, x, y \in S$, let $a^{\prime}$ be an inverse of $a, e=a a^{\prime}, s$ be an inverse of $a x y, D$ be the $D$-class of $S$ containing axy. Then axysaa' $\in E_{D^{\prime}}$ ayx, xya $\in D$ and there exists $u \in D$ such that ayxuxya $\in E_{D}$ since $D$ is completely simple. Further, $e \geq a x y s a a^{\prime}$ and $e \geq a y x u x y a^{\prime}$ which by the hypothesis yields axysaa' $=$ ayxuxya'. Consequently,

$$
a x y=a x y s a x y=\left(a x y s a a^{\prime}\right)(a x y)=\left(a y x u x y a^{\prime}\right)(a x y) \in a y x S
$$

and, dually, ayx $\in$ axyS. Hence axy $R$ ayx which shows that $S / R$ is a left normal band.

The statements concerning $\mathcal{Q}$ are established in an analogous manner.

(b) $\Rightarrow(c)$. Since $\mathcal{H}=\mathscr{L} \cap \mathcal{R}$, we have that $\mathcal{H}$ is a congruence and that $S / \mathcal{H}$ is a subdirect product of the right normal band $S / \mathscr{Q}$ and the left normal band $S / R$. Hence we may consider $S / \mathcal{H}$ as a subsemigroup of $(S / \mathscr{Q}) \times(S / \mathscr{R})$ and obtain 


$$
\begin{aligned}
\left(a_{1}, a_{2}\right)\left(x_{1}, x_{2}\right)\left(y_{1}, y_{2}\right)\left(a_{1}, a_{2}\right) & =\left(a_{1} x_{1} y_{1} a_{1}, a_{2} x_{2} y_{2} a_{2}\right) \\
= & \left(a_{1} y_{1} x_{1} a_{1}, a_{2} y_{2} x_{2} a_{2}\right)=\left(a_{1}, a_{2}\right)\left(y_{1}, y_{2}\right)\left(x_{1}, x_{2}\right)\left(a_{1}, a_{2}\right) .
\end{aligned}
$$

Thus $S / \mathcal{H}$ is a normal band.

(c) $\Rightarrow$ (d). First let $B$ be a normal band, let $a, b, c \in B$ with $a \leq b$. Then $a c=(a b) c=a(b c) c=(a c)(b c)$ and similarly $a c=(b c)(a c)$ so that $a c \leq b c$. Next

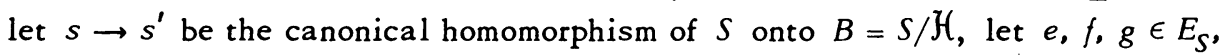
and suppose that $e \leq f$. Then $e^{\prime} \leq f^{\prime}$ and hence $e^{\prime} g^{\prime} \leq f^{\prime} g^{\prime}$ so that $(e g)^{\prime} \leq(f g)^{\prime}$. Consequently, $(e g)^{\prime}=(e g)^{\prime}(f g)^{\prime}=(f g)^{\prime}(e g)^{\prime}$ and thus

$$
(e g)^{*} \mathcal{H}(e g)^{*}(f g)^{*}, \quad(e g)^{*} \mathcal{H}(f g)^{*}(e g)^{*} .
$$

But then

$$
(e g)^{*}(f g)^{*}=\left[(e g)^{*}(f g)^{*}\right](e g)^{*}=(e g)^{*}\left[(f g)^{*}(e g)^{*}\right]=(f g)^{*}(e g)^{*}
$$

and hence both $(e g)^{*}(f g)^{*}$ and $(f g)^{*}(e g)^{*}$ are idempotents. It then follows from (1) that

$$
(e g)^{*}=(e g)^{*}(f g)^{*}=(f g)^{*}(e g)^{*},
$$

i.e. $(e g)^{*} \leq(f g)^{*}$. The proof of $(g e)^{*} \leq(g f)^{*}$ is dual.

(d) $\Rightarrow$ (a). Let $e, f, g \in E_{S}$ with $e \geq f, e \geq g, f \mathscr{D} g$. Then $g=g e=(g e)^{*} \geq$ $(g f)^{*}$ and similarly $g \geq(f g)^{*}$. Since $g, g f, f g$ are all $\mathscr{D}$-related, it follows that $g=$ $(g f)^{*}=(f g)^{*}$. Analogously $f=(g f)^{*}=(f g)^{*}$ which yields $f=g$.

$(a) \Rightarrow(e)$. We have seen in the preceding section that such a semigroup $S$ is a subdirect product of semigroups $\Pi\left(T_{D}\right)$ as $D$ ranges over all $D$-classes of $S$. Since for no $\mathscr{D}$-class $D$, the semigroup $D^{0}$ has zero divisors, the same holds for the semigroups $\Pi\left(T_{D}\right)$.

(e) $\Rightarrow$ (a). This follows from 3.4 .

Using 3.4 it is easy to see that the semigroups in 4.1 , and only they, can be constructed as follows (cf. [17, Theorem 6]; [6, 2.21, 2.22]).

Construction 4.2. Let $Y$ be a semilattice, for each $\alpha \in Y$, let $S_{\alpha}$ be a completely simple semigroup, and suppose that $S_{\alpha} \cap S_{\beta}=\varnothing$ if $\alpha \neq \beta$. For $\alpha, \beta \in Y$ with $\alpha>\beta$, let $\theta_{\alpha, \beta}: S_{\alpha} \rightarrow S_{\beta}$ be a homomorphism, let $\theta_{\alpha, a}$ be the identity mapping on $S_{\alpha}$, and suppose that

$$
\theta_{\alpha, \beta} \theta_{\beta, \gamma}=\theta_{\alpha, \gamma} \text { if } \alpha>\beta>\gamma \text {. }
$$

On the set $S=\bigcup_{a \in Y} S_{a}$ define a multiplication by

$$
a * b=\left(a \theta_{a, \alpha \beta}\right)\left(b \theta_{\beta, a \beta}\right) \quad\left(a \in S_{a}, b \in S_{\beta}\right) .
$$


Then $S$ satisfies the conditions of 4.1 , and conversely, every such semigroup can be obtained in this way.

For a given semilattice $Y$ and sets $S_{\alpha}$ with functions $\theta_{\alpha, \beta}$ as above, call the system $\left\{\theta_{\alpha, \beta}\right\}$ transitive if it satisfies (2).

Corollary 4.3 (cf. [4]). The following conditions on a completely regular semigroup $S$ are equivalent.

(a) $S$ satisfies the conditions 4.1 and $E_{S}$ is a subsemigroup of $S$.

(b) $E_{S}$ is a normal band.

(c) $S$ is a subdirect product of a class of semigroups of the following types: groups and left or right zero semigroups, and any of these with a zero adjoined.

Proof. (a) $\Rightarrow$ (c). By 4.1, $S$ is a subdirect product of semigroups $T$ and/or $T^{0}$ with $T$ completely simple, where now $E_{T}$ forms a subsemigroup of $T$ since $T$ or $T^{0}$ is a homomorphic image of $S$. But then $T$ is isomorphic to the direct product $G \times L \times R$ where $G, L, R$ are a group, and a left and a right zero semigroup, respectively (for a proof, see, e.g., [11, Lemme 1]). Hence $T^{0} \cong(G \times L \times R)^{0}$ where the latter is a subdirect product of semigroups $G^{0}, L^{0}, R^{0}$.

(c) $\Rightarrow(b)$. Since in each of the semigroups listed in (c) idempotents form a normal band, the same holds for any of their subdirect products.

(b) $\Rightarrow$ (a). By 4.1, Es satisfies $\mathfrak{D}$-majorization, which evidently implies that the same holds for $S$, and thus $S$ satisfies the conditions of 4.1.

It follows from (c) that subdirectly irreducible groups, 2-element left or right zero semigroups, and all of these with a zero adjoined constitute the class of all subdirectly irredicible semigroups satisfying the conditions of 4.3.

In order to give a construction of the semigroups satisfying the conditions of 4.3 , in the above construction we take $S_{\alpha}=G_{\alpha} \times L_{\alpha} \times R_{\alpha}$ and compute all homomorphisms of such a semigroup into another of the same type. This can be done by specializing the expression of a homomorphism of an arbitrary completely ( $0_{-}$) simple semigroup into another (see, e.g., [1, Theorem 3.11]) or by a direct consideration as in [12]. All homomorphisms $\theta: G \times L \times R \rightarrow G^{\prime} \times L^{\prime} \times R^{\prime}$ are given as follows: let $\omega: G \rightarrow G^{\prime}$ be a homomorphism, $\phi: L \rightarrow L^{\prime}$ and $\psi: R \rightarrow R^{\prime}$ be functions, and let $(g, l, r) \theta=(g \omega, l \phi, r \psi)$.

Construction 4.4. Let $Y$ be a semilattice, for each $\alpha \in Y$, let $G_{\alpha}$ be a group, $L_{\alpha}$ and $R_{\alpha}$ be nonempty sets, and suppose that $G_{\alpha} \cap G_{\beta}=L_{\alpha} \cap L_{\beta}=R_{\alpha} \cap R_{\beta}=\varnothing$ if $\alpha \neq \beta$. For $\alpha, \beta \in Y$ with $\alpha>\beta$, let $\omega_{\alpha, \beta}: G_{\alpha} \rightarrow G_{\beta}$ be a homomorphism, $\phi_{a, \beta}: L_{\alpha} \rightarrow L_{\beta}$ and $\psi_{a, \beta}: R_{\alpha} \rightarrow R_{\beta}$ be functions, let $\omega_{a, \alpha}, \phi_{a, a}, \psi_{a, a}$ be the identity functions on $G_{\alpha}, L_{\alpha}, R_{\alpha}$, respectively, and suppose that each of the systems $\left\{\omega_{a, \beta}\right\},\left\{\phi_{a, \beta}\right\},\left\{\psi_{a, \beta}\right\}$ is transitive. On the set $S=\bigcup_{\alpha \in Y}\left(G_{\alpha} \times L_{\alpha} \times R_{\alpha}\right)$ define a multiplication as follows: for $(g, l, r) \in G_{\alpha} \times L_{\alpha} \times R_{\alpha}$ and $\left(g^{\prime}, l^{\prime}, r^{\prime}\right) \epsilon$ $G_{\beta} \times L_{\beta} \times R_{\beta}$, 


$$
(g, l, r) *\left(g^{\prime}, l^{\prime}, r^{\prime}\right)=\left(\left(g \omega_{\alpha, \alpha \beta}\right)\left(g^{\prime} \omega_{\beta, \alpha \beta}\right), l \phi_{\alpha, \alpha \beta}, r^{\prime} \psi_{\beta, a \beta}\right) .
$$

Then $S$ satisfies the conditions of 4.3 , and conversely, every such semigroup can be obtained in this way.

This subject is related to the theme of the structure of regular semigroups satisfying permutational identities, see [16].

5. Conditions on ideals of a regular semigroup. We now return to regular semigroups and ask the following question: what can we say about ideals of a regular semigroup $S$ if $S$ satisfies $D$-majorization or $E_{S}$ is a tree? The theorems below give an answer to this question for $S$ suitably (and, indeed, strongly) restricted; the converse of these results is not true, but a partial converse will be established in $\$ 7$. To this end, we first introduce a new concept.

Definition 5.1. Let $I$ be an ideal of $S$ and $\sigma$ be a binary relation on a semigroup $S$. Then $I$ is a $\sigma$-categorical ideal of $S$ if, for any $a, b, c \in S, a b, b c \notin I$ and $a b \sigma b c$ imply $a b c \notin I$.

When $\sigma$ is the universal relation, a $\sigma$-categorical ideal is the usual categorical ideal; if $S$ has a zero which is a categorical ideal of $S$, then $S$ is said to be categorical at zero (see $[1, \$ 7.7]$, where $S$ itself is not a categorical ideal, we do not make this exception).

Lemma 5.2. The following conditions on an arbitrary semigroup $S$ are equivalent.

(a) For all $a, b, c \in S$, if $a b \mathrm{~g} b c$ then $a b c \mathcal{g} a b$ [either $a b c \mathrm{~g} a b$ or $a b c \mathscr{G} b c]$.

(b) All principal ideals of $S$ are 9 -categorical [categorical].

(c) All ideals of $S$ are I-categorical [categorical].

Proof. We will prove only the unbracketed statements, the bracketed statements are treated analogously.

(a) $\Rightarrow$ (b). If $a b, b c \notin J(x)$ and $a b \mathscr{I} b c$, then $a b c \mathscr{I} a b$ and thus $a b c \notin J(x)$.

(b) $\Rightarrow(c)$. Let $I$ be an ideal of $S$ and $a b, b c \notin I, a b I b c$. For every $x \in I$, the principal ideal $J(x)$ is $\mathcal{J}$-categorical and $J(x) \subseteq I$. Thus $a b, b c \notin J(x)$, and hence $a b g b c$ implies $a b c \notin J(x)$. Since $x \in I$ is arbitrary, it follows that $a b c$ $\notin I$ and $I$ is $I$-categorical.

(c) $\Rightarrow$ (a). If $a b \mathcal{I} b c$, then $a b, b c \notin I(a b)$ and since $I(a b)$ is either $\mathcal{G}$-categorical or empty, we must have $a b c \notin I(a b)$ so that $a b c \mathscr{g} a b$.

Theorem 5.3. If a regular semigroup $S=S^{0}$ satisfies (a) $\mathfrak{D}=\mathfrak{G}$, (b) all principal factors of $S$ are categorical at zero, (c) D-majorization, then all ideals of $S$ are D-categorical.

Proof. For any $D$-classes $A$ and $B$, if $A \leq B$, then the hypothesis implies 
$A \preceq B$, so by 2.3 the function $\eta_{B A}: B \rightarrow A$ satisfies $(\alpha)$ and $(\beta)$, and by 2.9 the system of functions $\left\{\eta_{B A}\right\}$ satisfies $(\epsilon)$. Now suppose that $a b \mathfrak{D} b c$. Letting $\alpha=\eta_{D_{a} D_{a b}}, \beta=\eta_{D_{b} D_{a b}}, \gamma=\eta_{D_{c} D_{a b}}$, we obtain $a b=(a \alpha)(b \beta), b c=(b \beta)(c \gamma)$. It follows that $a a, b \beta, c \gamma,(a \alpha)(b \beta),(b \beta)(c \gamma) \in D_{a b}$ where $D_{a b}$ can be considered as the set of all nonzero elements of the principal factor $J(a b) / I(a b)$. By hypothesis, the latter is categorical at zero which implies that $(a \alpha)(b \beta)(c \gamma) \in D_{a b}$. By $(\epsilon)$ we also have $b c=(b \beta) c$ and thus $a b c=(a \alpha)(b \beta) c=(a \alpha)(b \beta)(c \gamma) \in D_{a b}$. Hence $a b c \mathfrak{D} a b$ and 5.2 implies that all ideals of $S$ are $\mathscr{D}=\mathscr{I}$-categorical.

Recall that a partially ordered set $P$ is called a tree if, for any $a, b, c \in P$, the relations $a \geq b, a \geq c$ imply that either $b \geq c$ or $c \geq b$.

Theorem 5.4. If $S$ is a completely semisimple semigroup for which $E_{S}$ is a tree, then all ideals of $S$ are categorical.

Proof. By 5.2 it suffices to show that for any $a, b, c \in S$, we have $a b c \in D_{a b}$ $\cup D_{b c}$. Since $J(a b) \subseteq J(b)$ and $J(b c) \subseteq J(b)$, by 2.11 there exist idempotents $e \in D_{b}, f \in D_{a b}, g \in D_{b c}$ such that $e \geq f, e \geq g$. The hypothesis implies that either $f \geq g$ or $g \geq f$; suppose that $f \geq g$, the other case is treated analogously. Then $J(b c) \subseteq J(a b) \subseteq J(b)$ which by 2.11 and the hypothesis implies $D_{b c} \leq D_{a b^{\prime}}$ $D_{a b} \preceq D_{b}, D_{b c} \preceq D_{b}$. Now let $\phi=\eta_{D_{b} D_{a b}}, \psi=\eta_{D_{a b} D_{b c}}, \chi=\eta_{D_{b} D_{b c}}$, and let $b^{\prime}$ be an inverse of $b$. Using 2.3, 2.6, and 2.10, we obtain

$$
\begin{aligned}
(a b) \psi=\left(a b b^{\prime} b\right) \psi & =\left[\left(a b b^{\prime}\right)(b \phi)\right] \psi=\left(a b b^{\prime}\right) \psi(b \phi \psi)=\left(a b b^{\prime}\right) \psi(b \chi), \\
b c & =b\left(b^{\prime} b c\right)=(b \chi)\left(b^{\prime} b c\right),
\end{aligned}
$$

so that $\left(a b b^{\prime}\right) \psi, b \chi, b^{\prime} b c,\left(a b b^{\prime}\right) \psi(b \chi),\left(b_{\chi}\right)\left(b^{\prime} b c\right) \in D_{b c}$. Since $D_{b c}^{0}$ is completely 0-simple and thus categorical at zero, it follows that $\left(a b b^{\prime}\right) \psi(b \chi)\left(b^{\prime} b c\right) \in D_{b c}$ and (1) implies $(a b) \psi\left(b^{\prime} b c\right) \in D_{b c}$. Here $a b \in D_{a b^{\prime}}, b^{\prime} b c \in D_{b c}$, so by 2.5 we conclude that

$$
a b c=(a b)\left(b^{\prime} b c\right)=(a b) \psi\left(b^{\prime} b c\right) \in D_{b c}
$$

as desired.

If $S$ is an extension of a completely simple semigroup by a completely 0 -simple semigroup, then all ideals of $S^{0}$ are categorical but we cannot say anything about the idempotents of $S^{0}$. Thus the converses of both 5.3 and 5.4 fail badly.

6. Extensions of a primitive regular semigroup without contractions. In order to establish a converse of the theorems in the preceding section, we consider here a more general situation, namely that of extensions determined by partial homomorphisms of a primitive regular semigroup without contractions. For the pertinent material consult [1, Chapter 3].

Definition 6.1. A regular $\Lambda \times I$ matrix $P$ over a group with zero bas no contractions if 


$$
\begin{aligned}
& p_{\lambda i} \neq 0 \leftrightarrow p_{\mu i} \neq 0 \text { for every } i \in I \text { implies } \lambda=\mu, \\
& p_{\lambda i} \neq 0 \leftrightarrow p_{\lambda_{j}} \neq 0 \text { for every } \lambda \in \Lambda \text { implies } i=j .
\end{aligned}
$$

Thus $P$ has no contractions if and only if no two distinct rows and no two distinct columns of $P$ have the corresponding entries simultaneously different from zero. The case of $P$ without contractions may be considered as a weakening of the condition that $P$ be an invertible matrix and also as the opposite extreme of the case in which all entries of $P$ are different from zero.

Recall that a completely 0 -simple semigroup $T$ with trivial subgroups is called a rectangular $0-$ band, and that the congruence induced on an arbitrary semigroup $S$ by a homomorphism $\phi$ of $S$ onto $T$ is called an I-matrix congruence where $I=0 \phi^{-1}$ (see [7]). A congruence on a semigroup different from the universal relation is called proper. The next proposition will be quite useful.

Proposition 6.2. The following conditions on a regular Rees matrix semigroup. $S=\mathbb{R}^{0}(G ; I, \Lambda ; P)$ are equivalent.

(a) $P$ bas no contractions.

(b) $\mathcal{H}$ is the only 0-matrix congruence on $S$.

(c) Every proper congruence on $S$ is contained in $\mathcal{H}$.

(d) For $e, f \in E_{S}$, exe $\neq 0 \Leftrightarrow f x f \neq 0$ for all $x \in S$ implies $e=f$.

Proof. (a) $\Rightarrow($ b). By $[6,4.24]$ every proper congruence on $S$ is obtained from a triple $(N, p, \pi)$ where $N$ is a normal subgroup of $G, p$ and $\pi$ are partitions of $I$ and $\Lambda$, respectively. The conditions in $[6,4.22]$ on $p$ and $\pi$ in conjuction with our hypothesis imply that both must be the equality relations. On the other hand, the congruence associated with $(N, p, \pi)$ is a 0 -matrix congruence if and only if $N=G$. Consequently the only 0 -matrix congruence on $S$ is the one associated with $\left(G, i_{I}, \iota_{\Lambda}\right)$ where $i_{I}$ and $\iota_{\Lambda}$ are the equality relations on $I$ and $\Lambda$, respectively, namely $\mathcal{H}$.

(b) $\Rightarrow$ (c). By $[6,4.22,4.24]$ and the hypothesis, only the triples of the form $\left(N, i_{l}, \iota_{\mathbf{\Lambda}}\right)$ are associated with proper congruences on $S$, since otherwise $(G, p, \pi)$ for either $p \neq i_{I}$ or $\pi \neq \iota_{\mathbf{\Lambda}}$ would yield a 0 -matrix congruence different from $\mathcal{H}$. .Hence by $[6,4.25]$, every proper congruence on $S$ is contained in $\mathcal{H}$.

(c) $\Rightarrow$ (d). The relation $M$ defined on $S$ by

$$
a \Re b \text { if } a x a \neq 0 \leftrightarrow b x b \neq 0 \text { for all } x \in S
$$

is by $[7,2.6]$ the largest 0 -matrix congruence on $S$. Hence $\mathbb{M}$ is proper and thus the hypothesis implies $\mathbb{M} \subseteq \mathcal{H}$. Thus if $e, f \in E_{S}$ satisfy $e \mathbb{M} f$ we obtain $e \mathcal{H} f$ so that $e=f$.

(d) $\Rightarrow$ (a). Suppose that, for all $\lambda \in \Lambda, p_{\lambda i} \neq 0 \Leftrightarrow p_{\lambda j} \neq 0$. There exists $\mu \in \Lambda$ such that $p_{\mu_{i}} \neq 0$, so also $p_{\mu_{j}} \neq 0$. Hence for any $(x ; k, \lambda) \in S$, 


$$
\begin{aligned}
\left(p_{\mu i}^{-1} ; i, \mu\right)(x ; k, \lambda)\left(p_{\mu i}^{-1} ; i, \mu\right) \neq 0 & \Leftrightarrow p_{\mu k} \neq 0, p_{\lambda_{i}} \neq 0 \Leftrightarrow p_{\mu k} \neq 0, p_{\lambda j} \neq 0 \\
& \Leftrightarrow\left(p_{\mu j}^{-1} ; j, \mu\right)(x ; k, \lambda)\left(p_{\mu j}^{-1} ; j, \mu\right) \neq 0
\end{aligned}
$$

which by the hypothesis implies $\left(p_{\mu i}^{-1} ; i, \mu\right)=\left(p_{\mu j}^{-1} ; j, \mu\right)$ and hence $i=j$. One shows analogously that $p_{\lambda_{i}} \neq 0 \leftrightarrow p_{\mu_{i}} \neq 0$ for all $i \in I$ implies $\lambda=\mu$.

Even though the concept "no contractions" was defined for a sandwich matrix, the above proposition shows that for a completely 0 -simple semigroup $S$, this notion does not depend on the particular Rees matrix representation. For this reason, we say that $S$ bas no contractions and, more generally, introduce the following concept.

Definition 6.3. A completely semisimple semigroup $S=S^{0}$ bas no contractions if every nonzero principal factor of $S$ is without contractions (i.e., satisfies any of the conditions in 6.2 ). If $S$ is completely semisimple without zero, then $S$ bas no contractions if $S^{0}$ has none.

Proposition 6.4. Every completely semisimple semigroup $S$ without contractions bas the property that every congruence on $S$ contained in $\mathcal{D}$ is also contained in $\mathcal{H}$.

Proof. Let $\sigma$ be a congruence on $S$ such that $\sigma \subseteq \mathfrak{D}$, and let $P=J(a) / I(a)$ be a principal factor of $S$. If $I(a)=\varnothing$, then $P$ is the kernel of $S$ and the hypothesis implies that $P$ must be a group, which then trivially implies that $\left.\left.\sigma\right|_{P} \subseteq \mathcal{H}\right|_{P}$. Suppose next that $I(a) \neq \varnothing$. Since $P^{*}=J(a) \backslash I(a)$, the restriction $\left.\sigma\right|_{P^{*}}$ is an equivalence relation on $P^{*}$ which can be extended to a congruence $\tau$ on $P$ by letting the zero of $P$ be $\tau$-related only to itself. Hence $\tau$ is a proper congruence of the completely 0 -simple semigroup $P$ which by 6.2 implies that $\tau$ is contained in the $\mathcal{H}$ equivalence on $P$. Since the restriction of the $\mathcal{H}$ equivalence on $S$ to $P^{*}$ coincides with the restriction to $P^{*}$ of the $\mathcal{H}$ equivalence on $P$, and $P$ is an arbitrary principal factor of $S$, it follows that $\sigma \subseteq \mathcal{H}$, the $\mathcal{H}$ equivalence on $S$.

The converse of 6.4 holds if for every principal factor $P$ of $S$, every proper congruence on $P$ restricted to $P^{*}$ can be extended to a congruence on $S$ contained in $\mathscr{D}$. This occurs in the case we consider next. Recall that a semigroup $S=S^{0}$ is an orthogonal sum of (its sub-) semigroups $S_{\alpha}, \alpha \in A$, if $S=\bigcup_{a \in A} S_{\alpha}$, $S_{\alpha} S_{\beta}=S_{\alpha} \cap S_{\beta}=0$ if $\alpha \neq \beta$ (0-direct union in [1, $\left.\$ 6.3\right]$ ); the semigroups $S_{\alpha}$ are called components. We will write $S=\Sigma_{\alpha \in A} \bigoplus S_{\alpha}$, and if there are only two components, $S=S_{1} \oplus S_{2}$. A regular semigroup $S=S^{0}$ is called primitive if all its nonzero idempotents are primitive; these are precisely the semigroups which are orthogonal sums of completely 0 -simple semigroups $[7,5.16]$.

Corollary 6.5. The following conditions on a primitive regular semigroup $S$ are equivalent. 
(a) $S$ bas no contractions.

(b) Every congruence on $S$ which is contained in $\mathfrak{D}$ is also contained in $\mathcal{H}$.

(c) For $e, f \in E_{S}$, exe $\neq 0 \Leftrightarrow f x f \neq 0$ for all $x \in S$ implies $e=f$.

Proof. (a) $\Rightarrow($ b). This is a special case of 6.4 .

(b) $\Rightarrow(c)$. Since $S$ is an orthogonal sum of completely 0 -simple semigroups, it follows without difficulty that the relation $M$ defined in the proof of 6.2 (c) $\Rightarrow$ (d) is a congruence on $S$ contained in $\mathfrak{D}$ and thus also in $\mathcal{H}$, which implies that (c) holds.

(c) $\Rightarrow(a)$. Condition (c) then also holds in each completely 0-simple component of $S$ and hence 6.2 implies (a).

Recall that an ideal $I$ of a semigroup $S$ is prime if for any $a, b \in S, a S b \subseteq I$ implies that either $a \in I$ or $b \in I$, and that $I$ is a matrix ideal if it is both prime and categorical (see [7] where categorical is called quasi-complètement premier).

Lemma 6.6. Let $V=V^{0}$ be a semigroup categorical at zero with an ideal $S=$ $\Sigma_{a \in A} \oplus S_{a}$ where each $S_{\alpha}$ is completely 0-simple. Then $V=\left(\Sigma_{a \in A} \oplus T_{a}\right) \oplus Q$ where, for every $a \in A$, we bave $S_{\alpha} \subseteq T_{\alpha}$ and 0 is a matrix ideal of $T_{\alpha}$.

Proof. Let $T=\{v \in V \mid S v \neq 0\} \cup 0$ and $Q=(V \backslash T) \cup 0$. If $s \in S$ and $v \in V$ are such that $s v \neq 0$, then $s v=(s v) u(s v)$ for some $u \in S$ since $s v \in S$ and $S$ is regular. But then $u s \in S$ and $v(u s) \neq 0$. By symmetry, we conclude that $S v \neq 0$ $\Leftrightarrow v S \neq 0$, and the definition of $T$ is actually symmetric. If $t \in T, v \in V$, and $t v \neq 0$, then $s t \neq 0$ for some $s \in S$ and hence the hypothesis implies that $s t v \neq 0$, so that $t v, v \in T$. It follows similarly that $t \in T, v \in V$, and $v t \neq 0$ imply $v t, v$ $\epsilon T$. Consequently, $T$ is an ideal of $V$ and, for any $t \in T, q \in Q$, we have $t q=$ $q t=0$. If $x, y \in V$ and $x y \in T^{*}$, then $s x y \neq 0$ for some $s \in S$ and thus $x, y \in T$. It follows by contraposition that $Q$ is a subsemigroup of $V$, which then shows that $V=T \oplus Q$, and by construction, $S \subseteq T$.

If $t \in T^{*}$, then $s t \neq 0$ for some $s \in S$ and hence $s t=(s t) u(s t)$ for some $u \epsilon$ $S$, so that $t T t \neq 0$. In such a case, 0 is called a semiprime ideal of $T$ and since 0 is also a categorical ideal of $T[7,5.13]$ implies that $T=\Sigma_{\beta \in B} \oplus T_{\beta}$ where 0 is a matrix ideal of each $T_{\beta}$. It is easy to see that each $S_{\alpha}$ is an ideal of $S$ and thus also of $T$. Since 0 is a prime ideal of each $T_{\beta}$, if $s_{1} \in S_{a_{1}}^{*}, s_{2} \in S_{a_{2}}^{*}$, and $s_{a_{1}} \cup S_{a_{2}} \subseteq T_{\beta}$, then for some $t \in T_{\beta}, s_{1} t s_{2} \neq 0$. But $s_{1} t s_{2} \in S_{\alpha_{1}}^{*} \cap s_{a_{2}}^{*}$ and hence $\alpha_{1}=\alpha_{2}$. Consequently, each $T_{\beta}$ contains at most one $S_{\alpha}$. If $t \in T_{\beta}^{*}$ then $s t \in S$ for some $s \in S_{\alpha}^{*}$ for some $\alpha$ which shows that $S_{\alpha} \subseteq T_{\beta}$ and each $T_{\beta}$ contains at least one $S_{\alpha}$. Therefore we may label the $T_{\beta}$ 's in such a way that $S_{\alpha}$ is contained only in $T_{a}$.

We will take for granted definitions and simple properties of an (ideal) extension, partial homomorphism, an extension determined by a partial homomorphism, and inflation (see $[1, \$ 4.4]$ and [3]). The next theorem and its corollaries were 
established in [15] for the special case of a primitive inverse semigroup. It seems remarkable that in it and its corollaries the nature of the entire extension is dictated by its behavior at zero.

Theorem 6.7. An extension $V$ of a primitive regular semigroup $S$ without contractions is determined by a partial homomorphism if and only if $V$ has a categorical ideal I such that I $\cap S=0$.

Proof. Necessity. The proof is the same as the proof of necessity of [15, Theorem 1] and does not require the hypothesis of no contractions.

Sufficiency. As in the proof of sufficiency of [15, Theorem 1], the problem can be reduced to the case $I=0$. By $[7,5.16]$ we can write $S=\Sigma_{a \in A} \bigoplus S_{a}$ where each $S_{a}$ is completely 0 -simple, so by 6.6 we obtain $V=\left(\Sigma_{a \in A} \oplus T_{a}\right) \oplus Q$ where, for each $\alpha \in A, S_{\alpha}$ is a 0 -minimal ideal of $T_{\alpha}$ and 0 is a matrix ideal of $T_{\alpha}$. Now the restriction of the smallest 0 -matrix congruence $\Phi_{a}$ on $T_{a}$ to $S_{\alpha}$ is a 0 -matrix congruence on $S_{\alpha}$. Since $S_{\alpha}$ has no contractions, 6.2 implies that the $\mathcal{H}$ equivalence $\mathcal{H}_{\alpha}$ on $S_{\alpha}$ is its only 0 -matrix congruence, which then implies that $\left.\Phi_{\alpha}\right|_{s_{\alpha}}=$ $\mathcal{H}_{a}$. Hence $[7,4.15 . \mathrm{I}]$ is satisfied and thus $[7,4.15 . \mathrm{II}]$ yields the existence of a homomorphism $\phi_{\alpha}$ mapping $T_{\alpha}$ onto $S_{\alpha}$ and leaving the latter elementwise fixed. Defining $\phi$ on all of $V$ by $v \phi=v \phi_{\alpha}$ if $v \in T_{\alpha}$ and $v \phi=0$ if $v \in Q$, it follows at once that $\phi$ is a homomorphism of $V$ onto $S$ leaving the latter elementwise fixed. By [13, Proposition 2], $V$ is an extension determined by a partial homomorphism (in fact, by $\left.\phi\right|_{V \backslash s}$ ).

The proofs of the following corollaries are the same as those of the corollaries to [15, Theorem 1].

Corollary 6.8. A semigroup $V$ is an inflation of a primitive regular semigroup without contractions if and only if $V$ is categorical at zero and $V^{2}$ is a primitive regular semigroup without contractions.

Corollary 6.9. Let $S$ be a primitive regular semigroup without contractions and let $T$ be a semigroup categorical at zero and having no nonzero proper ideals. Then an extension $V$ of $S$ by $T$ is determined by a partial homomorphism if and only if $V$ is categorical at zero.

7. Completely semisimple semigroups without contractions. We will prove in this section that for this class of semigroups the converses of 5.3 and 5.4 are valid. These results have several interesting consequences. We begin by characterizing the property "no contractions" in terms of elements of a completely semisimple semigroup.

Proposition 7.1. A completely semisimple semigroup $S$ bas no contractions if and only if, for any $e, f \in E_{S}$, 


$$
e \in \operatorname{Sexe} S \Leftrightarrow f \in S f x f S \text { for all } x \in S \text { implies } e=f \text {. }
$$

Proof. Necessity. Let $e, f \in E_{S}$ and suppose that, for all $x \in S, e \in$ SexeS $\Leftrightarrow f \in S f x f S$. Since $e \in \operatorname{Seee} S$, we have $f \in S f e f S \subseteq S e S=J(e)$ and hence $J(f) \subseteq$ $J(e)$; by symmetry, we also have $J(e) \subseteq J(f)$. Letting $P=J(e) / I(e)$, we obtain $e, f \in P^{*}$. Let $x \in P$ and suppose that exe $\neq 0$ in $P$. Since $P$ is completely (0-)simple, $e=$ uexev for some $u, v \in P^{*}$. Consequently, $e \in$ SexeS since $u, x$, $v \in S$ and the hypothesis implies that $f \in S f x f S$, that is, $f=a f x f b$ for some $a, b$ $\epsilon S$. It follows that $f x f \in P^{*}$ which proves that $f x f \neq 0$ in $P$. By symmetry, we conclude that, for all $x \in P$, exe $\neq 0 \Leftrightarrow f x f \neq 0$ which by 6.2 yields $e=f$.

Sufficiency. First let $P$ be a completely 0 -simple semigroup and suppose that, for $e, f \in E_{P}$, we have exe $\neq 0 \Leftrightarrow f x f \neq 0$ for all $x \in P$. If $x e y \neq 0$, then $x e \neq 0$ and $e y \neq 0$, so there exists $u \in P$ such that eyuxe $\neq 0$. The hypothesis then implies that fyuxf $\neq 0$ and thus $f y \neq 0$ and $x f \neq 0$ so that $x f y \neq 0$. By symmetry, we conclude that $x e y \neq 0 \leftrightarrow x f y \neq 0$ for all $x, y \in P$.

Now let $P$ be a principal factor of $S$, let $e, f \in E_{P^{*}}$ and suppose that, for all $x \in P$, exe $\neq 0 \Leftrightarrow f x f \neq 0$. Suppose next that $e \in$ SeyeS for some $y \in S$. Then $e=a e y e b$ for some $a, b \in S$ and hence $e a, y e b e \in P$ and $(e a) e(y e b e) \neq 0$ in $P$. By the above, $(e a) f(y e b e) \neq 0$ in $P$. Since eafy, be $\in P$, again by the above, (eafy) $f(b e) \neq 0$ in $P$. Consequently $f \in P^{*}$ eafyfbe $P^{*} \subseteq S f y f S$; by symmetry, we conclude that for all $y \in S, e \in$ SeyeS $\Leftrightarrow f \in S f y f S$, which by the hypothesis yields $e=f$. Therefore $P$ has no contractions.

The next result provides a partial converse of 5.3 .

Theorem 7.2. The following conditions on a completely semisimple semigroup $S$ without contractions are equivalent.

(a) $S$ satisfies D-majorization.

(b) All ideals of $S$ are $D$-categorical.

(c) $S$ is a subdirect product of completely 0-simple semigroups without contractions.

Proof. (a) $\Rightarrow(\mathrm{c})$. As we have seen in the proof of $3.4, S$ is a subdirect product of its principal factors, which here by the overall hypothesis have no contractions.

(c) $\Rightarrow($ a). This is a part of 3.4 and holds in any completely semisimple semigroup.

(a) $\Rightarrow(b)$. This is a special case of 5.3 .

(b) $\Rightarrow$ (a). Let $e, f, g \in E_{S}$ and suppose that $e \geq f, e \geq g, f \mathfrak{D} g$. Let $P=$ $J(f) / I(f)$ and suppose that $f x f \neq 0$ in $P$ for some $x \in P$. Then $f \operatorname{exf} \neq 0$ and hence ex $\in P^{*}$. Consequently, ge, ex $\notin I(f)$ and $g e \mathfrak{D}$ ex and thus the hypothes is implies that $g$ ex $\notin I(f)$ (if $I(f)=\varnothing$, this is trivially satisfied). Hence in $P, g x$ 
$\neq 0$, and an analogous argument shows that also $x g \neq 0$. But then $g x g \neq 0$; by symmetry, we conclude that, for all $x \in P, f x f \neq 0 \Leftrightarrow g x g \neq 0$. Since $P$ has no contractions, it follows that $f=g$.

The next corollary should be compared with 3.4 .

Corollary 7.3. The following conditions on a semigroup $S$ are equivalent.

(a) $S$ is a completely semisimple inverse semigroup satisfying $D$-majorization.

(b) $S$ is a completely semisimple inverse semigroup all of whose ideals are D-categorical.

(c) $S$ is regular and a subdirect product of Brandt semigroups.

The next theorem is a partial converse of 5.4.

Theorem 7.4. In a completely semisimple semigroup $S$ without contractions all of whose ideals are categorical, $E_{S}$ is a tree.

Proof. Let $e, f, g \in E_{S}$ and suppose that $e \geq f, e \geq g$. If $J(f) \neq J(f g) \neq J(g)$, then $f e, e g \notin J(f g)$ and the hypothesis implies that $f g=f e g \notin J(f g)$, a contradiction. By symmetry, we may suppose that $J(g)=J(f g)$ so that $J(g) \subseteq J(f)$. If $I(g)=\varnothing$, then the hypothesis implies that $J(g)=J_{g}$ is a group, and $J(g) \subseteq J(f)$ by 2.11 yields $f \geq g$ since $g$ is the only idempotent in $J_{g} \cdot$

Suppose next that $I(g) \neq \varnothing$. Since $I(g)$ is a categorical ideal in $S$, we obtain that the semigroup $V=J(e) / I(g)$ is categorical at zero. Further, $T=$ $J(g) / I(g)$ is a completely 0 -simple semigroup without contractions and an ideal of $V$. Letting $I=0$ in 6.7, we conclude that $V$ is an extension of $T$ determined by a partial homomorphism $\phi$. Hence the hypothesis $e \geq g$ implies that $e \phi \geq g$, but since $T$ is completely 0 -simple, we must have $e \phi=g$. Again by 2.11 , the hypothesis $J(g) \subseteq J(f)$ implies the existence of $b \in E_{J_{g}}$ such that $f \geq b$. But then $e \geq$ $f \geq b$ which as above implies that $e \phi=b$. Consequently, $f \geq b=e \phi=g$ as desired.

Corollary 7.5. In a completely semisimple semigroup $S$ without contractions, $E_{S}$ is a tree if and only if all ideals of $S$ are categorical.

In particular, the conclusion is valid in any completely semisimple inverse semigroup; this is precisely the content of [15, Theorem 2].

Proposition 7.6. If $S$ is a regular semigroup in which $\mathfrak{D}=\mathcal{G}$ and all of whose ideals are categorical, then $S / \mathcal{G}$ is a tree.

Proof. Suppose that $J(a) \supseteq J(b)$ and $J(a) \supseteq J(c)$. By 2.11 , there exist $f \in$ $E_{J_{b}}$ and $g \in E_{J_{c}}$ such that $e \geq f$ and $e \geq g$. Since then $f e g=f g \in J(f g)$, the hypothesis implies that either $f \in J(f g)$ or $g \in J(f g)$. Consequently, either $J(f)=$ $J(f g)$ or $J(g)=J(f g)$, so that either $J(b) \subseteq J(c)$ or $J(c) \subseteq J(b)$ and thus $S / \mathcal{G}$ is a tree. 


\section{REFERENCES}

1. A. H. Clifford and G. B. Preston, The algebraic theory of semigroups. Vols. I, II, Math. Surveys, vol. 7, Amer. Math. Soc., Providence, R. I., 1961, 1967. MR 24 \#A2627; MR 36 \#1558.

2. N. Farès, Idempotents et D-classes dans les demi-groupes et les anneaux, C. R. Acad. Sci. Paris Sér. A-B 269 (1969), A341-A343. MR 40 \#2777.

3. P. A. Grillet and M. Petrich, Ideal extensions of semigroups, Pacific J. Math. 26 (1968), 493-508. MR 38 \#5967.

4. N. Kimura, Note on idempotent semigroups. III, Proc. Japan Acad. 34 (1958), 113-114. MR $20 \# 4604$.

5. B. P. Kočin, The structure of inverse ideal-simple $\omega$-semigroups, Vestnik Leningrad. Univ. 23 (1968), no. 7, 41-50. (Russian) MR 37 \#2881.

6. G. Lallement, Demi-groupes réguliers, Ann. Mat. Pura Appl. (4) 77 (1967), 47129. MR $37 \# 1505$.

7. G. Lallement and M. Petrich, Décompositions I-matricielles d'un demi-groupe, J. Math. Pures Appl. (9) 45 (1966), 67-117. MR 33 \#5765.

8. - Structure d'une classe de demi-groupes réguliers, J. Math. Pures Appl. (9) 48 (1969), 345-397. MR 41 \#3638.

9. E. S. Ljapin, Semigroups, Fizmatgiz, Moscow, 1960; English transl., Transl. Math. Monographs, vol. 3, Amer. Math. Soc., Providence, R. I., 1968. MR 22 \#11054. \#5316.

10. W. D. Munn, Regular $\omega$-semigroups, Glasgow Math. J. 9 (1968), 46-66. MR 37

11. M. Petrich, Sur certaines classes de demi-groupes. I, Acad. Roy. Belg. Bull. Cl. Sci. (5) 49 (1963), 785-798. MR 29 \#3560.

12. - Kongruenzen einiger spezieller vollständig einfacher Halbgruppen, Math. Nachr. 32 (1966), 73-81. MR $35 \# 272$.

13. - On extensions of semigroups determined by partial homomorphisms,

Nederl. Akad. Wetensch. Proc. Ser. A $69=$ Indag. Math. 28 (1966), 49-51. MR 33 \#5775.

14. - Representations of semigroups and the translational hull of a regular Rees matrix semigroup, Trans. Amer. Math. Soc. 143 (1969), 303-318. MR 40 \#253.

15. - On a class of completely semisimple inverse semigroups, Proc. Amer. Math. Soc. 24 (1970), 671-676. MR 41 \#372.

16. M. Yamada, Regular semigroups whose idempotents satisfy permutation identities, Pacific J. Math. 21 (1967), 371-392. MR 37 \#2887.

17. M. Yamada and N. Kimura, Note on idempotent semigroups. II, Proc. Japan Acad. 34 (1958), 110-112. MR 20 \#4603.

DEPARTMENT OF MATHEMATICS, PENNSYLVANIA STATE UNIVERSITY, UNIVERSITY

PARK, PENNSYLVANIA 16802 